\title{
Reflexo econômico e desenvolvimento da resistência de Phakopsora pachyrhizi a fungicidas em função do número de aplicações
}

\author{
Erlei Melo Reis ${ }^{1}$, Andrea Camargo Reis ${ }^{2}$ e Mateus Zanatta ${ }^{2}$
}

${ }^{1}$ Programa de Pós-graduação da Universidade de Buenos Aires, Av. San Martin 4453 - C1417DSE - Buenos Aires - Argentina. ${ }^{2}$ Agroservice Pesquisa e consultoria agrícola - Passo Fundo, RS, Brasil

Autor para correspondência: Erlei Melo Reis (erleireis@upf.br)

Data de chegada: 24/03/2017. Aceito para publicação em: 04/10/2017.

$10.1590 / 0100-5405 / 176382$

\section{RESUMO}

Reis, E.M; Reis, A.C.; Zanatta, M. Reflexo econômico e desenvolvimento da resistência de Phakopsora pachyrhizi a fungicidas em função do número de aplicações. Summa Phytopathologica, v.44, n.3, p.289-292, 2018.

O objetivo do controle de doenças de plantas com fungicidas é minimizar os danos causados à cultura, incluindo a redução de produção. No entanto, o uso de fungicidas impacta no custo de produção e aumenta o risco de seleção de linhagens de fungos resistentes. Esses dois fatores são dependentes do número de pulverizações. Atualmente, no uso de fungicidas na cultura da soja visando, principalmente, ao controle da ferrugem (Phakopsora pachyrhizi) não se adota qualquer critério científico, para definir quando efetuar a primeira aplicação. Nessa revisão é abordada a importância do monitoramento das lavouras de soja, da diagnose e da quantificação de danos causados pela ferrugem. Apresentam-se os principais indicadores empíricos para a primeira aplicação e o critério científico. A ocorrência da resistência cruzada e múltipla de P. pachyrhizi aos fungicidas sítio específicos carboxamidas, estrobilurinas e triazóis pode ter sido acelerada pelo uso desnecessário de aplicações desses fungicidas.

Palavras-chave: Ferrugem da soja, controle químico, Glycine max, limiar de dano econômico.

\section{ABSTRACT}

Reis, E.M; Reis, A.C.; Zanatta, M. Economic repercussion and fungicide resistance development in Phakopsora pachyrhizi dependent on the number of applications. Summa Phytopathologica, v.44, n.3, p.289-292, 2018.

The objective of plant disease control using fungicides is to minimize the damage caused to the crop, including yield damage. However, the use of fungicides affects the production cost and increases the risk of selection of resistant fungal strains. These two factors are dependent on the number of fungicide sprayings. Currently, the use of fungicides in soybean crops with the particular aim of controlling Asian soybean rust (Phakopsora pachyrhizi) has not adopted any scientific criteria to define when the first application should be done. This review addresses the importance of soybean crop monitoring, as well as of disease diagnosis and quantification of damages caused by soybean rust. The main empirical indicators for the first application and the scientific criterion are presented. The occurrence of cross and multiple resistance in P. pachyrhizi to the site-specific fungicides carboxamides, strobilurins and triazoles may have been accelerated by the unnecessary number of fungicide applications.

Keywords: Asian soybean rust, chemical control, Glycine max, economic damage threshold.

A afirmativa de Main (19) de que os agricultores cultivam a terra para ganhar dinheiro merece profunda análise. Dentro desse contexto a aplicação de fungicidas e, portanto, o número total de aplicações, deve ser feito com racionalidade à luz da ciência assegurando o retorno econômico de sua aplicação, pois envolve custos (3, 20, 21).

O foco dessa revisão é discutir a relação entre o número de aplicações de fungicidas e a sustentabilidade econômica e ambiental, tomando como exemplo a ferrugem da soja, causada pelo fungo basidiomiceto Phakopsora pachyrhizi Sydow \& Sydow (35).

Algumas perguntas pertinentes ao tema ajudam melhor entendelo: qual o número necessário de aplicações que deve ser feita para a obtenção do lucro máximo? Quais os princípios que norteiam a tomada de decisão de aplicar ou não fungicida?

Considerando-se a estratégia antirresistência (16), o número de aplicações deve ser discutido. Relatos confirmam relação entre o número de pulverizações e o desenvolvimento da resistência (10). Os fungicidas não são agentes mutagênicos, mas sim selecionadores. A ciência tem mostrado como calcular o número correto de aplicações para a obtenção de controle econômico. Se a primeira aplicação for feita na ausência da doença pode ameaçar a economia e aumentar o risco do desenvolvimento da resistência aos fungos alvo do controle. Assim, o FRAC (10) alerta de que quanto maior o número de aplicações maior a pressão de seleção direcional. A vida efetiva dos fungicidas sítio específicos deve ser mantida pelo maior tempo possível.

Se a ferrugem da soja, não causasse dano e não reduzisse o lucro, esta revisão não faria sentido. Sabendo-se o preço da tonelada da soja, o custo da aplicação de fungicidas/ha, o coeficiente de dano (relação da severidade com o rendimento de grãos), o potencial produtivo da lavoura e a eficiência do controle se pode determinar a necessidade ou não da aplicação de fungicida resultando ou não em retorno econômico $(4,7,23,30,37)$.

A importância do tema é reforçada ao considerar-se a área cultivada com soja na safra 2016/17, carca de 33,7 milhões de hectares (6), custo médio de uma aplicação de R \$352.00/ha, ou $301 \mathrm{~kg}$ de soja/ha (preço da soja de $\mathrm{R} \$ 70.00 / 60 \mathrm{~kg}$ em fevereiro de 2017) (3).

Os danos causados pelas doenças são qualitati- e quantitativos 
$(17,20,24)$. Nas culturas produtoras de grãos, como a soja, são mais importantes os quantitativos. Por outro lado, os qualitativos são importantes em horti- fruticultura. Em consequência, um número maior de aplicações de fungicidas são necessárias para o controle dos qualitativos.

\section{Manejo integrado de doenças (MID)}

O MID consiste na "utilização de todas as técnicas disponíveis dentro de um programa unificado de tal modo a manter a população de organismos nocivos (doenças) abaixo do limiar de dano econômico (LDE) e a minimizar os efeitos colaterais deletérios ao meio ambiente" (22). Infelizmente esses princípio não têm sido considerados no emprego de fungicidas!

Quando o controle é reduzido pelo desenvolvimento da resistência, também é reduzido o lucro dos produtores.

No exemplo de controle da ferrugem da soja, devem ser considerados três princípios: (i) garantir o controle econômico; (ii) reduzir a seleção direcional do fungicida em direção do desenvolvimento da resistência; (iii) reduzir os efeitos deletérios no ambiente considerando a oferta da sociedade pela produção de alimentos mais limpos. Os três são importantes, mas os dois primeiros são mais facilmente percebidos.

Como o foco do tema é o número de aplicações de fungicidas em soja, os critérios disponíveis indicadores do momento para se fazer a primeira aplicação devem ser discutidos em detalhe por que resulta diretamente no número de aplicações e no custo de sua aplicação.

Monitoramento de lavouras. A definição do momento para a primeira aplicação, requer o monitoramento, não pode ser feita à distância. Para a obtenção do controle a presença inicial da doença (intensidade que não causa dano) o período de proteção não é aproveitado.

Conceito. O monitoramento consiste em visitas periódicas na lavoura durante todo o seu ciclo $(12,13,15,18,34)$. Um dos principais objetivos tem sido determinar a necessidade ou não da aplicação de fungicidas. No entanto, é comum entre os assistentes técnicos a pergunta o que é monitoramento, como é feito e para que serve? Por essas e outras razões o monitoramento das lavouras de soja tem sido ignorado e não utilizado. Outras perguntas, igualmente importantes, podem ser acrescentadas: Qual é a utilidade do conhecimento em fitopatologia (métodos de amostragem de lavouras, técnicas de diagnose, quantificação de doenças e principalmente estimar seus danos)? É reconhecida a importância desse tema pelos Agrônomos e usados na cultura da soja ou em outras culturas? Qual é o valor de publicações que contém imagens das doenças da soja? Os agricultores exigem tais conhecimentos dos consultores responsáveis técnicos?

Utilidade do monitoramento. Sua importância decorre da diagnose correta e em estimar os danos causados pela doença alvo do controle. O dano causado depende da intensidade e não da simples presença da doença. Para isso foram estabelecidas as funções de dano relacionando a severidade com o dano. Portanto, a tomada de decisão em aplicar fungicida ou não depende da quantidade do dano $(5,18,34)$.

Quantificação da doença. O monitoramento envolve a fitopatometria, técnica de quantificar a intensidade da doença e estimar danos causados $(5,7,17)$. É a intensidade da doença que determina a necessidade ou não da aplicação de medidas de controle. A intensidade das doenças pode ser mensurada pela incidência ou severidade em órgãos (ex. folíolos da soja).

Para a tomada de decisão, foram estabelecidos níveis ou limiares relacionando a intensidade da doença com o dano causado $(2,21,37)$. $\mathrm{O}$ dano econômico é função direta da quantidade da doença e não de sus presença. Desse modo, doenças que não causam dano econômico, ou quando as que causam não atingem o limiar, não devem ser alvo da aplicação de fungicidas segundo o princípio do MID. Sem o monitoramento (diagnose e quantificação de dano), não se pode tomar decisão embasada em conhecimento científico para aplicar ou não fungicidas!

Critérios indicadores do momento para a primeira aplicação de fungicidas em soja. Critérios são referências que indicam a necessidade da aplicação de fungicidas. Critério é uma regra que permite a fundamentação racional de uma escolha, é uma decisão.

Critérios empíricos. Empirismo é o conhecimento adquirido pela experiência ou pela observação prática e não pelo método científico.

Estádio fenológico. O estádio fenológico não é um fator determinante de doença $(1,11)$, mas tem sido por muito tempo tomado como orientador da primeira aplicação visando ao controle da ferrugem da soja, como por exemplo o estádio R1 (estádio de florescimento) (31). Ultimamente tem sido recomendado aos produtores aplicarem fungicida em estádios vegetativos como V4 e V5. Pode se observar que a primeira aplicação tem sido feita cada vez mais cedo. Segundo esse critério, a presença, a intensidade da ferrugem e o dano não são considerados; sendo por isso sempre necessária a aplicação $(21,22,28)$. Em geral, resulta em aplicação desnecessária que ameaçam a lucratividade e aumentando a seleção direcional. Na maioria das vezes, resulta em falta de proteção da cultura no final do ciclo.

Pré-fechamento das linhas da lavoura de soja. Os recomendantes desse critério o justificam que uma maneira de melhor a deposição do fungicida na camada inferior do dossel da lavoura é a aplicação nesse momento (26). Com o lançamento de cultivares de soja de arquitetura ereta, permanecendo com a folhagem parcialmente afastada mantenho pequeno espaço entre fileiras até o final do ciclo. Portanto, esse critério não resiste ao argumento da melhora da deposição, pois essa alegação pode ser resolvida pelo cultivo de cultivares com essa caraterística desejável. No entanto, se for observado esse critério, sempre será necessária a aplicação, mas seguramente sendo desnecessária, sem retorno econômico, pois a doença não está presente. Os recomendantes desse indicador, reforçam de sua importância como sendo fundamental na primeira aplicação, esquecem-se das demais aplicações. É lógica a pergunta, por que então na segunda e demais aplicações esse critério não é mais válido? Agora não há necessidade de deposição na parte inferior do dossel? $\mathrm{Na}$ ausência da doença valia e agora em sua presença não mais? Se conclui que sempre, independentemente da presença ou da intensidade da doença o agricultor sempre terá que aplicar fungicida. Aplicações desnecessárias aumentam o custo e contribuem para acelerar a seleção direcional à resistência dos fungicidas sítio específicos.

Critério preventivo. Mais recentemente, um indicador inovativo para a primeira aplicação - o chamado momento preventivo- tem sido proposto aos sojicultores (25). Por conceito, é a aplicação do fungicida feita na completa ausência da ferrugem, sensu Hewitt (14). É mais um indicador apontando que, independentemente da presença/intensidade da doença, a aplicação sempre deverá ser feita. No entanto, muitos consultores perguntam como esse momento pode ser identificado numa lavoura? Novamente, como os anteriores, esse indicador resulta em aplicações desnecessárias ao controle da ferrugem. A justificativa para o uso desse critério é de que as estrobilurinas agem principalmente no processo de germinação dos esporos a aplicação deve ser feita antes da deposição dos esporos. Merece ser discutido que quando as estrobilurinas forem aplicadas na segunda e demais aplicações, e na realidade o são, a justificativa é esquecida agora apresentando ação protetora, curativa e erradicativa.

Deve ser lembrado que os fungicidas penetrantes móveis 
apresentam ação protetora, curativa e erradicativa $(12,30)$.

Critério científico. A literatura científica apresenta poucas alternativas técnicas como suporte científico e racionalmente fundamentada, para a primeira aplicação, de fungicidas numa $(2,7$, $9,21,22,36,37)$.

Considerando a importância do manejo integrado de doenças, a literatura científica apresenta, como critério racional para a primeira aplicação, o nível ou limiar de dano econômico $(2,7,9,21,22,36$, 37). Tem como base identificar a intensidade de doença que causa dano econômico. Portanto, quando a intensidade causar dano econômico, antes desse valor for ser alcançado, o fungicida deve ser aplicado (limiar de ação). Nesse caso a seleção direcional deve ser minimizada.

Por que os critérios empíricos tem sido os mais difundidos entre os produtores? Por outro lado, por que o critério cientifico - MID sustentável, não é largamente difundido e usado pelos agricultores? O critério científico serve apenas para países desenvolvidos com agricultura mais tecnificada ou produtores mais esclarecidos?

O período de proteção e o número de aplicação de fungicidas. O período de proteção de um fungicida é o número de horas, ou de dias, que a concentração inibitória do fungicida permanece nas folhas tratadas resultando no controle eficiente $(>80 \%)$ da doença. Ultimamente, aceita-se em 15 dias o período de proteção conferido pelos fungicidas penetrantes móveis em soja. Ao final desse período, não há mais concentração suficiente nos tecidos foliares para conferir proteção, sendo necessária uma nova aplicação imediata.

Assim, o número de aplicações depende: (i) do ciclo da cultivar de soja; (ii) do momento da primeira aplicação; (iii) da dose que quanto menor mais curto o período de proteção do fungicida; e (iv) da duração do período de proteção. Por essas razões o número de aplicações é variável. Quanto mais longo for o período de proteção menor será o número de aplicações $\mathrm{O}$ inverso é verdadeiro. Hoje, pelo desenvolvimento da resistência de $P$. pachyrhizi, o período de proteção dos fungicidas tem sido reduzido (de 21 para $<$ de 15 dias) exigindo num menor tempo maior concentração do fungicida no tecido foliar.

O número de aplicações de fungicidas e a seleção direcional para a resistência de fungos a fungicidas. Em adição ao fator econômico, o número de aplicações aumenta a seleção direcional FRAC (10). Quanto maior o número de aplicações mais intensa é a seleção direcional eliminado os indivíduos sensíveis e aumentando a população dos resistentes ao fungicida sítio específico. O número de aplicações aumenta em função do mau posicionamento incorreto da primeira, requerendo na maioria dos casos, uma aplicação adicional no fim do ciclo da cultura. Sendo a primeira, indicada pelo LDE e observando-se o intervalo de 15 dias entre as aplicações seria a direção correta a ser seguida.

O FRAC internacional não recomenda mais do que duas aplicações/ área/safra de fungicidas sítio específicos (IDM, IQe e ISDH) (10). Por isso, a primeira aplicação deve ser estrategicamente posicionada. Infelizmente essa regra não tem sido observada em relação ao controle químico da ferrugem da soja no Brasil, e, portanto, a seleção direcional tem sido acelerada safra após safra.

O número de aplicações de fungicida pode reduzir o lucro dos produtores, poluir o ambiente e acelera a seleção direcional em direção da resistência do fungo as sítio específicos reduzindo seu período de vida efetivo. Essa é a ameaça à sustentabilidade econômica da sojicultora.

Os fungicidas penetrantes móveis têm ação preventiva, curativa e erradicativa à $P$. pachyrhizi $(10,22)$. Assim, a primeira aplicação pode ser feita seguramente segundo o limiar de dano econômico $(4,21)$.
As novas cultivares de soja com arquitetura que mantém as fileiras parcialmente abertas durante todo o seu ciclo, permitem a deposição dos fungicidas na parte inferior do dossel melhorando assim a eficiência do controle. Tais cultivares deverão ser mais utilizadas pelos produtores.

O excessivo número de aplicações de fungicidas tem aumentado a seleção direcional em direção da seleção de indivíduos resistentes aos fungicidas sítio específicos $(16,20)$. É preocupante a evolução da redução do controle da ferrugem da soja, safra-após-safra.

Devido ao uso de fungicidas sítio específicos, isolados ou em misturas duplas ou triplas, com o número descontrolado de aplicações, nas próximas safras, haverão fungicidas com controle eficiente $(>80 \%)$ para o controle da ferrugem da soja? Essa é a maior ameaça que deverá ser enfrentada nas próximas safras de soja.

É possível reduzir o número de aplicações de fungicidas observando-se a semeadura de cultivares de soja precoces no início da época recomendada, observar o vazio sanitário, eliminar plantas voluntárias e adotar limiar de dano econômico na tomada de decisão para a primeira aplicação (33).

\section{REFERÊNCIAS}

1. Agrios, J.N. Plant pathology. San Diego: Elsevier Academic Press, 2005, $5^{\text {th }}$ ed. 922 p.

2. Bergamin Filho, A.; Amorim, L. Doenças de plantas tropicais: epidemiologia e controle econômico. São Pulo: Agronômica Ceres, 1996. 289 p.

3. Boller, W. Aspectos econômicos da aplicação de fungicidas em órgãos aéreos. In: Reis, E. M. Org. Critérios indicadores do momento para a primeira e intervalo de aplicações de fungicidas nas culturas de soja e trigo. Passo Fundo, Aldeia Norte Editora. 2010.

4. Casa, R.T.; Blum, M.M.C.B.; Danelli, A.L. Critério limiar de dano econômico. In: Reis, E.M. Indicadores do momento para a aplicação de fungicidas visando ao controle de doenças nas culturas da soja e do trigo. Berthier: Passo Fundo, p. 109-132, 2013.

5. Chester, K. S. Plant disease losses: their appraisal and interpretation. Plant Disease Reporter Supplement, St. Paul, v.193, p.191-362, 1950.

6. Conab. Acompanhamento grãos da safra brasileira. Safra 2016/17 - N.5, v. 4 Safra 2016/17 - Quinto levantamento, Brasília, p. 1-166 fevereiro 2017. ISSN 2318-6852.

7. Danelli, A. L. D.; Reis, E. M.; Boaretto, C. Critical-point model to estimate yield loss caused by Asian soybean rust. Summa Phytopathologica, Botucatu, v. 41, n. 4, p. 262-269, 2015.

8. FAO. Report of the first session of the F.A. O. Panel of experts on integrated pest control. F.A. O. Meeting Report. No. PL/1967/M/7. Annals, Rome.

9. Fawcet, H. S.; Lee, H. A. Citrus diseases and their control. McGraw-Hill, New York. 1926

10. FRAC. Fungicide resistance action committee. Global Crop Protection Organization. Brussels, 2012. Disponível em: www.gcpt.org/frac.

11. Furtado, G. Q.; Alves, S. A. M.; Carneiro, L.C.; Godoy, C. V.; Masspla Júnior, N. S. Influência do estádio fenológico e da idade dos trifólios de soja na infecção de Phakopsora pachyrhizi. Tropical Plant Pathology, Brasília, vol. 34, 2, 118-122, 2009.

12. Godoy, C.V.; Canteri, M.G. Efeitos protetor, curativo e erradicante de fungicidas no controle da ferrugem da soja causada por Phakopsora pachyrhizi, em casa-de-vegetação. Fitopatologia Brasileira, Brasília, v.29, p.97-101, 2004.

13. Gregg, B.R.; Camargo, C.P.; Popinigis, F.; Lingerfelt, C.W.; Vechi, C. Guia de inspeção de campos para produção de sementes. Brasília. Ministério da Agricultura. AGIPLAN, 1974.98p.

14. Hewitt, H. G. Fungicides in crop protection. CAB International, 1998. Chapter 4. Fungicide performance. P. 87- 15

15. Holshouse, D.; Elizabeth Bush, Phipps, P.; Stromberg, E. Extension. Asian Soybean Rust - Frequently Asked Questions V: Monitoring, Tracking, and Scouting. Virginia Cooperative Extension. Publication 450-305. https:// www.pubs.ext.vt.edu/content/dam/pubs_ext_vt_edu/450/450-305/450305 pdf.pdf 
16. Ishii, H.; Hollomon, D.W. Fungi resistance in plant pathogens. Principles and guide to practical management. Springer Japan, 2015, 490p.

17. Kranz, J. Measuring plant disease. In Experimental techniques in plant disease epidemiology. J. Kranz, \& J. Rotem, ed. Springer-Verlag, Heidelber. p. $35-50,1988$.

18. Madden, L.V.; Hugh, G. Sampling for plant disease incidence. symposium Sampling for Decision Making in Crop Loss Assessment and Pest Management , St. Paul, v. 89 (11), p.1088-1103, 1999.

19. Main, C.E. Crop destruction - the raison d'être of plant pathology. In: Horsfall, J.G. \& Cowling, E.B. (Ed.) Plant disease an advance treatise. How disease is managed. New York. Academic Press. pp 55-78. 1977.

20. McCarl, B.A. Economics of integrated pest management: An interpretive review of the literature. Oregon Agricultural Experiment Station. Spec. Rep. 636, 142p. 1981

21. Munford, J. D.; Norton, G. A. Economics of decision making in pest management. Ann. Rev. Entomology, Palo Alto, 29:157-174.1984.

22. NAS. Insect pest management and control. Public. 1695. National Academy of Sciences, Washington. 1969.

23. Nutter, F., Esker, P., and Coelho-Netto, R. Disease assessment concepts and the advancements made in improving the accuracy and precision of plant disease data. Iowa State University. European Journal of Plant Pathology, Dordrecht, v. 115, p.95-103, 2006.

24. Nutter, F.W. Jr, Teng, S.P. \& Royer, M.H. Terms and concepts for yield, crop, and disease thresholds. Plant Disease, St. Paul, v.77, p. 211-215, 1993.

25. Reis, E.M. Critério preventivo. In: Reis, E.M. Indicadores do momento para a aplicação de fungicidas visando ao controle de doenças nas culturas da soja e do trigo. Berthier: Passso Fundo, p. 67-76, 2013.

26. Reis, E.M. Critério pré-fechmaneto da soja. In: Reis, E.M. Indicadores do momento para a aplicação de fungicidas visando ao controle de doenças nas culturas da soja e do trigo. Berthier: Passo Fundo, p. 77-87, 2013.

27. Reis, E.M. Critério início da doença. In: Reis, E.M. Indicadores do momento para a aplicação de fungicidas visando ao controle de doenças nas culturas da soja e do trigo. Berthier: Passo Fundo, p. 95-107. 2013.

28. Reis, E.M. Critério estádio fenológico da planta. In: Reis, E.M. Indicadores do momento para a aplicação de fungicidas visando ao controle de doenças nas culturas da soja e do trigo. Berthier: Passo Fundo, p. 95-107, 2013.

29. Reis, E. M.; Silva, L.H.C.P.; Siqueri, F.V.; Silva, J.R.C. Evolução da redução da sensibilidade de Phakopsora pachyrhizi a fungicidas e estratégia para recuperar a eficiência do controle. Berthier: Passo Fundo, 2. ed. rev. e atual., 2015. 103p.

30. Reis, E.M.; Zanatta, M.; Reis, Andrea, C. Eficiência do controle de uma doença para igualar ao custo da aplicação terrestre de fungicida. Um exemplo de cálculo com a ferrugem da soja. Summa Phytopathologica, Botucatu, v. p. Carta ao Editor. (in press).

31. Reis, E. M.; Zanatta, T.; Zanatta, M. Curative and eradicant action of fungicides to control Phakopsora pachyrhizi in soybean plants. Summa Phytopathologica, Botucatu, v.42, n.4, p.295-302, 2016.

32. Ritchie, S.; Hanway, J.J.; Thompson, H.E., 1982. How a soybean plant develops. Ames, Iowa State University of Science and Technology, Coop. Ext. Serv. 20p. (Special Report, 53).

33. Reunião de Pesquisa da Soja da Região Sul, Indicações Técnicas para a Cultura da Soja no Rio Grande do Sul e em Santa Catarina, safras 2012/2013 e 2013/2014. Passo Fundo: Embrapa Trigo. p.142, 2012.

34. Sasckatchewan Ministry of Agriculture. Plant Disease Scouting 101. June 2010. http://publications.gov.sk.ca/documents/20/84068-e9ab3e26-4ba9-4716-8504-330fe6cfbac7.pdf

35. Sydow, H.; Sydow, P. A contribution to knowledge of parasitic fungi of the island of Formosa. Annales Mycologici, v. 12, p. 108, 1914.

36. Stern, V.M.; Smith, R.F.; van den Bosch, R.; Hagen, K.S. The integrated control concept. Hilgardia, Berkley, v. 28, p.81-101. 1959.

37 Zadoks, J. C. On the conceptual basis of crop loss assessment: the threshold theory. Annual Review of Phytopathology, Palo Alto, 23:455-473. 1985. 